\title{
From transposon to chromosome and polyploidy. An update on cytogenetics and genomics of Arabidopsis
}

\author{
Martin A. Lysak • Paul F. Fransz
}

Published online: 13 June 2014

(C) Springer Science+Business Media Dordrecht 2014

$\begin{array}{ll}\text { Abbreviations } \\ \text { 3C } & \text { Chromosome conformation capture } \\ \text { 4C } & \text { Circularized chromosome conformation } \\ & \text { capture } \\ \text { cenH3 } & \text { Centromere-specific histone H3 } \\ \text { DSB } & \text { Double-strand break } \\ \text { Hi-C } & \text { Genome conformation capture technique } \\ \text { HR } & \text { Homologous recombination } \\ \text { LINC } & \text { Linker of nucleoskeleton and cytoskeleton } \\ \text { NGS } & \text { Next-generation sequencing } \\ \text { NHEJ } & \text { Non-homologous end joining } \\ \text { RdDM } & \text { RNA-directed DNA methylation } \\ \text { SAC } & \text { Spindle assembly checkpoint } \\ \text { smRNA } & \text { Small RNA } \\ \text { TE } & \text { Transposable element } \\ \text { WGD } & \text { Whole-genome duplication }\end{array}$

In 2003, Chromosome Research published a special issue on Arabidopsis as a cytogenetic model edited by Hans de Jong (Wageningen University). Historically,

\footnotetext{
Responsible Editor: Beth A Sullivan

M. A. Lysak $(\bowtie)$

Central European Institute of Technology (CEITEC),

Masaryk University, Kamenice 5, 62500 Brno,

Czech Republic

e-mail: martin.lysak@ceitec.muni.cz

\section{P. F. Fransz}

Swammerdam Institute for Life Sciences, University of Amsterdam, Science Park 904, 1098 XH Amsterdam, The Netherlands e-mail: P.F.Fransz@uva.nl
}

this was the first monothematic compendium on chromosome and interphase chromatin organization, meiosis, cytogenetic tools, and resources of the model plant Arabidopsis thaliana (Arabidopsis) and its close relatives. As pointed out by de Jong (2003), Arabidopsis "was considered to be unfavorable for cytogenetic research due to its tiny metaphase chromosomes and small interphase nuclei". However, this view was swept away by the release of the Arabidopsis genome sequence (Arabidopsis Genome Initiative 2000) and introduction of improved fluorescence-based protocols in plant cytogenetics. In the decade following the appearance of the first Arabidopsis special issue, we have seen further rapid development and diversification of chromosome research in Arabidopsis, brassicas, and several other Brassicaceae species. This progress was driven particularly, but not exclusively, by the advent of nextgeneration sequencing (NGS) technologies (Lister et al. 2009), the growing appreciation of non-coding RNAs, broader application of fluorescent-protein tagging systems, and the use of chromosome conformation capture technologies (3C, $4 \mathrm{C}$ and $\mathrm{Hi}-\mathrm{C})$ to study the architecture and folding principles of the entire chromosome complement during interphase (Moissiard et al. 2012; Grob et al. 2013). The present special issue, although providing only a snapshot of the current chromosome research on Arabidopsis and its close relatives, aims to summarize some of the most important achievements over the past decade and outline future research directions.

After expensive and laborious sequencing of the Arabidopsis genome in 2000, it was generally expected that the sequenced Columbia genome would become the 
gold standard of Arabidopsis-related research for many years to come. Jesse Hollister explains why this did not happen, walking us through the timeline of the "NGS revolution" and summarizing the major milestones in Arabidopsis genomics during the last decade. NGSenabled genomics paved the way for an ambitious endeavor of sequencing over a thousand Arabidopsis strains (1001 Genomes Project), to reveal the extent of structural genomic variation across the range of the species. This and other (re)sequencing efforts, along with genome sequencing in closely related species (Arabidopsis lyrata, Capsella rubella), make the genus Arabidopsis and the family Brassicaceae an emerging model system for comparative and evolutionary plant genomics.

Although the extant Arabidopsis genome is functionally diploid, whole-genome duplications (WGD) played a key role in the evolution of this species and the entire mustard family. Kirsten Bomblies and Andreas Madlung provide an extensive review of the incidence of auto- and allotetraploids in the genus Arabidopsis. They discuss that due to a wealth of genomic data for Arabidopsis and its congeners, the Arabidopsis genus serves as a powerful system to study epigenetic and molecular mechanisms following WGD events. In addition to the allotetraploid Arabidopsis suecica and autotetraploid Arabidopsis lines, forerunners in polyploidy research, autopolyploids Arabidopsis arenosa and A. lyrata, and the allopolyploid Arabidopsis kamchatica are now gaining increased attention. Genomic analyses of auto- and allopolyploid Arabidopsis taxa provide novel insights into molecular mechanisms of genome stabilization after polyploidization and better understanding of how a long-term survival of these polyploid species is attained.

Centromeres are crucial chromosomal loci ensuring regular chromosome segregation during mitosis and meiosis. Arabidopsis is one of the most important model species for studying the structure and function of eukaryotic centromeres. Inna Lermontova et al. provide a timely review of centromere research in Arabidopsis and other crucifer species, concentrating on the assembly and function of the kinetochore. They describe the essential role of the centromere-specific histone H3 (cenH3) in kinetochore assembly, tissue specificity of cen $\mathrm{H} 3$ expression, and recent developments in the regulation of cenH3 centromere loading. In addition, they discuss the diversity and function of Arabidopsis spindle assembly checkpoint (SAC) proteins that control correct attachment of kinetochores to microtubules of the spindle.

Whereas centromeres are essential for chromosome replication and segregation, telomeres guard the integrity of a chromosome by protecting its ends from being recognized as double-strand breaks (DSB). Arabidopsis is the most prominent model species in plant telomere research. Andrew Nelson et al. compare the telomeric repeat sequence, telomere length, and telomere-binding proteins among Arabidopsis and several other Brassicaceae species. They found that despite the conserved sequence of the telomere (TTTAGGG) across the crucifer species analysed, the telomere length varies considerably, even between homeologous chromosome arms. The paper also shows that telomere-binding proteins differ in copy number among the analysed species and that some genes remain duplicated while others exist as single copies. Nelson et al. set the stage in the field for follow-up studies on telomere evolution in Brassicaceae, a plant group that has provided the most mature understanding of telomere biology.

In contrast to the centromere, the telomere appears to be non-essential for the eukaryote chromosome as evidenced by circular chromosomes. Minoru Murata provides an overview of naturally occurring and experimentallyinduced linear and circular minichromosomes in Arabidopsis. Historically, minichromosomes have been analysed and manipulated to reveal essential functional structures of eukaryotic chromosomes, whereas contemporary efforts have been predominantly directed towards the construction of plant synthetic chromosomes. The recently produced artificial ring minichromosome AtARC1, meiotically transmissible to the next generation, is discussed in detail, particularly in the context of application of engineered chromosomes for improving crop varieties (see also Lermontova et al. 2014).

Catalytic events during meiosis are well conserved between plants and other eukaryotes. In their review, Eugene Sanchez-Moran and Sue Armstrong describe the recent progress of meiosis research in Arabidopsis in comparison to other species, focusing on key proteins that control the formation of crossovers. Instrumental to our knowledge of meiosis is the development of microscopic techniques, as well as genetic and molecular approaches. Progress in forward and reverse genetic approaches to investigate meiotic recombination is discussed.

Meiotic homologous recombination is a highly specialized mechanism to repair DSBs induced at the onset 
of meiosis. In fact, only a fraction of the induced DSBs is repaired via reciprocal crossovers. The majority of DSBs is repaired following other DNA repair pathways. Alexander Knoll et al. continue the theme of DSB repair in Arabidopsis, but with a focus on DNA repair pathways in somatic cells. They discuss mechanisms of DSB repair via non-homologous end joining (NHEJ) and homologous recombination (HR). Each DSB repair pathway exhibits distinct molecular patterns and can result in small genomic changes or even large chromosomal rearrangements, such as translocations of chromosome arms. This review emphasizes that how DSBs are repaired can have a major impact on genome integrity and evolutionary adaptation.

Another major source of genome rearrangement are the transposable elements (TEs), of which there are numerous in large eukaryote genomes. Transposons can move around in the genome via different interruption, copy, and insertion mechanisms. The review by Zoé Joly-Lopez and Thomas Bureau focuses on TEs that provide beneficial effects to host plants and their role in the formation of novel genes and regulatory networks. They also point out the importance of careful and proper annotation of TEs, an issue that impacts the gene-regulatory role of TEs in many organisms during development and in response to external cues. The involvement of TEs in transcription factor activity makes this group of genomic elements an exciting topic for future genome studies.

The review by Hidetaka Ito and Tetsuji Kakutani on transposon control in Arabidopsis complements the article by Joly-Lopez and Bureau. They summarize TE regulation via transcriptional and posttranscriptional silencing mechanisms controlled by DNA methylation. RNA-directed DNA methylation (RdDM) involves de novo methylation of TEs by DRM2 via small interfering RNAs. The review further describes the effect of TE control on developmental genes in plants and under stress conditions. How TEs shape and (re)organize the chromosomes is also discussed.

The past decade has witnessed a burst of information on non-coding transcripts that control genome function and chromosome stability. Small RNAs (smRNA) are the topic of the article by Pedro Costa-Nunes et al. This group presents an overview of the regulatory role of non-coding RNAs, including the molecular mechanism underlying RdDM. In particular, they address the spatial organization of smRNA processing and activities in the nucleus and provide insight into cellular compartmentalization and the transport of smRNAs and RdDM pathway members to and from the nucleus and cytoplasm.

Trafficking of molecules across the nuclear membrane requires protein networks in the nuclear envelope such as the nuclear pore complex, but also other protein complexes. The LINC (Linker of nucleoskeleton and cytoskeleton) complex at the inner and outer membrane of the nuclear envelope is the topic of the article by Christophe Tatout et al. They focus on two specific families, SUN and KASH domain proteins, that together form a physical link between the cytoskeleton and the nucleoskeleton across the nuclear envelope. The SUN domain protein family is well conserved across eukaryotes. LINC complex proteins are possibly involved in the formation of the nuclear envelope by specific chromatin connections. The identification of novel LINC proteins at the nuclear envelope in Arabidopsis is very exciting. Considering the close association of heterochromatic chromocenters at the nuclear membrane in Arabidopsis, it is tempting to speculate that LINC proteins play a role in chromatin silencing at the nuclear envelope.

As this second Arabidopsis issue has been a collective effort, we like to acknowledge the enthusiasm and outstanding contributions of all participating authors, and express our thanks to Beth A. Sullivan, Executive Editor of Chromosome Research, for her support and guidance through the whole editorial process.

\section{References}

Arabidopsis Genome Initiative (2000) Analysis of the genome sequence of the flowering plant Arabidopsis thaliana. Nature 408:796-815

De Jong H (2003) Foreword. Chromosom Res 11:181-182

Grob S, Schmid MW, Luedtke NW, Wicker T, Grossniklaus U (2013) Characterization of chromosomal architecture in Arabidopsis by chromosome conformation capture. Genome Biol 14:R129

Lermontova I, Sandmann M, Demidov D (2014) Centromere and kinetochores of Brassicaceae. Chromosome Res. doi:10. 1007/s10577-014-9422-z

Lister R, Gregory BD, Ecker JR (2009) Next is now: new technologies for sequencing of genomes, transcriptomes, and beyond. Curr Opin Plant Biol 12:107-118

Moissiard G, Cokus SJ, Cary J, Feng S, Billi AC, Stroud H, Husmann D, Zhan Y, Lajoie BR, McCord RP et al (2012) MORC family ATPases required for heterochromatin condensation and gene silencing. Science 336 : 1448-1451 